\title{
INEQUALITIES ON THE SPECTRAL RADIUS, OPERATOR NORM AND NUMERICAL RADIUS OF HADAMARD WEIGHTED GEOMETRIC MEAN OF POSITIVE KERNEL OPERATORS
}

\author{
ALJOŠA PEPERKO ${ }^{1,2}$
}

\begin{abstract}
Recently, several authors have proved inequalities on the spectral radius $\rho$, operator norm $\|\cdot\|$ and numerical radius of Hadamard products and ordinary products of non-negative matrices that define operators on sequence spaces, or of Hadamard geometric mean and ordinary products of positive kernel operators on Banach function spaces. In the present article we generalize and refine several of these results. In particular, we show that for a Hadamard geometric mean $A^{\left(\frac{1}{2}\right)} \circ B^{\left(\frac{1}{2}\right)}$ of positive kernel operators $A$ and $B$ on a Banach function space $L$, we have

$$
\rho\left(A^{\left(\frac{1}{2}\right)} \circ B^{\left(\frac{1}{2}\right)}\right) \leq \rho\left((A B)^{\left(\frac{1}{2}\right)} \circ(B A)^{\left(\frac{1}{2}\right)}\right)^{\frac{1}{2}} \leq \rho(A B)^{\frac{1}{2}} .
$$
\end{abstract}

In the special case $L=L^{2}(X, \mu)$ we also prove that

$$
\left\|A^{\left(\frac{1}{2}\right)} \circ B^{\left(\frac{1}{2}\right)}\right\| \leq \rho\left(\left(A^{*} B\right)^{\left(\frac{1}{2}\right)} \circ\left(B^{*} A\right)^{\left(\frac{1}{2}\right)}\right)^{\frac{1}{2}} \leq \rho\left(A^{*} B\right)^{\frac{1}{2}} .
$$

\section{INTRODUCTION}

In [25], X. Zhan conjectured that, for non-negative $n \times n$ matrices $A$ and $B$, the spectral radius $\rho(A \circ B)$ of the Hadamard product satisfies

$$
\rho(A \circ B) \leq \rho(A B),
$$

where $A B$ denotes the usual matrix product of $A$ and $B$. This conjecture was confirmed by K.M.R. Audenaert in [3] by proving

$$
\rho(A \circ B) \leq \rho^{\frac{1}{2}}((A \circ A)(B \circ B)) \leq \rho(A B) .
$$

These inequalities were established via a trace description of the spectral radius. Using the fact that the Hadamard product is a principal submatrix of the Kronecker product, R.A. Horn and F. Zhang proved in [14] the inequalities

$$
\rho(A \circ B) \leq \rho^{\frac{1}{2}}(A B \circ B A) \leq \rho(A B) .
$$

Applying a technique of [14]), Z. Huang proved that

$$
\rho\left(A_{1} \circ A_{2} \circ \cdots \circ A_{m}\right) \leq \rho\left(A_{1} A_{2} \cdots A_{m}\right)
$$

2010 Mathematics Subject Classification. 15A60, 47B34, 15A42, 47A10, 47B65.

Key words and phrases. Hadamard-Schur weighted geometric mean; Spectral radius; Operator norm; Numerical radius; Positive kernel operators; Banach function spaces; Non-negative matrices; Banach sequence spaces. 
for $n \times n$ non-negative matrices $A_{1}, A_{2}, \cdots, A_{m}$ (see [15]). The author of the current paper extended the inequality (1.3) to non-negative matrices that define bounded operators on Banach sequence spaces in [20]. Additional refinements of this inequality were proved by D. Chen and Y. Zhang in [6] and by R. Drnovšek and the author in [11], where they also obtained related inequalities for the operator norm and numerical radius. In the proofs of [20] and [11] certain results on the Hadamard product from [9] and [18] were used.

Earlier, A.R. Schep was the first one to observe that the results of [9] and [18] are applicable in this context (see [22] and [23]). In particular, in [22, Theorem 2.8] he proved that the inequality

$$
\rho\left(A^{\left(\frac{1}{2}\right)} \circ B^{\left(\frac{1}{2}\right)}\right) \leq \rho(A B)^{\frac{1}{2}}
$$

holds for positive kernel operators on $L^{p}$ spaces. Here $A^{\left(\frac{1}{2}\right)} \circ B^{\left(\frac{1}{2}\right)}$ denotes the Hadamard geometric mean of operators $A$ and $B$. This inequality was generalized in [11, Theorem 3.1], where it was shown that the inequality

$$
\rho\left(A_{1}^{\left(\frac{1}{m}\right)} \circ A_{2}^{\left(\frac{1}{m}\right)} \circ \cdots \circ A_{m}^{\left(\frac{1}{m}\right)}\right) \leq \rho\left(A_{1} A_{2} \cdots A_{m}\right)^{\frac{1}{m}}
$$

holds for positive kernel operators $A_{1}, \ldots, A_{m}$ on an arbitrary Banach function space. In [21], the author generalized the inequality (1.5) to the setting of the joint and generalized spectral radius.

In [15] and [6] the following upper bounds for the operator norm

$$
\|A \circ B\| \leq \rho\left(\left(A^{T} B\right) \circ\left(B^{T} A\right)\right)^{\frac{1}{2}} \leq \rho\left(A^{T} B\right)
$$

were proved for $n \times n$ non-negative matrices and these inequalities were extended to non-negative matrices that define operators on $l^{2}$ in [11].

The article is organized as follows. In the second section we introduce some definitions and facts, and we recall some results from [9] and [18], which we will need in our proofs. In Section 3 (Theorems 3.1 and 3.2 and Corollary 3.3) we generalize and refine inequality (1.5) for positive kernel operators on Banach function spaces and prove related inequalities for the operator norm and numerical radius. In particular, we refine inequality (1.4) in the following way (Corollary $3.5)$ :

$$
\rho\left(A^{\left(\frac{1}{2}\right)} \circ B^{\left(\frac{1}{2}\right)}\right) \leq \rho\left((A B)^{\left(\frac{1}{2}\right)} \circ(B A)^{\left(\frac{1}{2}\right)}\right)^{\frac{1}{2}} \leq \rho(A B)^{\frac{1}{2}}
$$

which can be seen as a kernel version of (1.2). In Theorem 3.6 we prove more general inequalities than (1.3), which are valid for non-negative matrices that define operators on Banach sequence spaces. In Section 4 we prove additional results for positive kernel operators on $L^{2}(X, \mu)$. In particular, in Theorem 4.4 we prove that the inequalities

$$
\left\|A^{\left(\frac{1}{2}\right)} \circ B^{\left(\frac{1}{2}\right)}\right\| \leq \rho\left(\left(A^{*} B\right)^{\left(\frac{1}{2}\right)} \circ\left(B^{*} A\right)^{\left(\frac{1}{2}\right)}\right)^{\frac{1}{2}} \leq \rho\left(A^{*} B\right)^{\frac{1}{2}}
$$

hold for such operators. In Theorem 4.6 and Remark 4.7 we generalize this result to several operators and we obtain an additional closely related result for nonnegative matrices that define operators on $l^{2}$ in Theorem 4.8. 


\section{Preliminaries}

Let $\mu$ be a $\sigma$-finite positive measure on a $\sigma$-algebra $\mathcal{M}$ of subsets of a non-void set $X$. Let $M(X, \mu)$ be the vector space of all equivalence classes of (almost everywhere equal) complex measurable functions on $X$. A Banach space $L \subseteq$ $M(X, \mu)$ is called a Banach function space if $f \in L, g \in M(X, \mu)$, and $|g| \leq|f|$ imply that $g \in L$ and $\|g\| \leq\|f\|$. Throughout the paper we will assume that $X$ is the carrier of $L$, that is, there is no subset $Y$ of $X$ of strictly positive measure with the property that $f=0$ a.e. on $Y$ for all $f \in L$ (see [24]).

Standard examples of Banach function spaces are Banach sequence spaces (explicitly defined bellow), the well-known spaces $L^{p}(X, \mu)(1 \leq p \leq \infty)$ and other less known examples such as Orlicz, Lorentz, Marcinkiewicz and more general rearrangement-invariant spaces (see e.g. [5], [7] and the references cited there), which are important, e.g., in interpolation theory. Recall that the cartesian product $L=E \times F$ of Banach function spaces is again a Banach function space, equipped with the norm $\|(f, g)\|_{L}=\max \left\{\|f\|_{E},\|g\|_{F}\right\}$.

By an operator on a Banach function space $L$ we always mean a linear operator on $L$. An operator $A$ on $L$ is said to be positive if it maps nonnegative functions to nonnegative ones, i.e., $A L_{+} \subset L_{+}$, where $L_{+}$denotes the positive cone $L_{+}=$ $\{f \in L: f \geq 0$ a.e. $\}$. Given operators $A$ and $B$ on $L$, we write $A \leq B$ if the operator $B-A$ is positive.

Recall that a positive operator $A$ on $L$ is always bounded, i.e., its operator norm

$$
\|A\|=\sup \left\{\|A x\|_{L}: x \in L,\|x\|_{L} \leq 1\right\}=\sup \left\{\|A x\|_{L}: x \in L_{+},\|x\|_{L} \leq 1\right\}
$$

is finite. Also, its spectral radius $\rho(A)$ is always contained in the spectrum.

In the special case $L=L^{2}(X, \mu)$ we can define the numerical radius $w(A)$ of a bounded operator $A$ on $L^{2}(X, \mu)$ by

$$
w(A)=\sup \left\{|\langle A f, f\rangle|: f \in L^{2}(X, \mu),\|f\|_{2}=1\right\} .
$$

If, in addition, $A$ is positive, then it is straightforward to prove that

$$
w(A)=\sup \left\{\langle A f, f\rangle: f \in L^{2}(X, \mu)_{+},\|f\|_{2}=1\right\} .
$$

From this it follows easily that $w(A) \leq w(B)$ for all positive operators $A$ and $B$ on $L^{2}(X, \mu)$ with $A \leq B$.

An operator $A$ on a Banach function space $L$ is called a kernel operator if there exists a $\mu \times \mu$-measurable function $a(x, y)$ on $X \times X$ such that, for all $f \in L$ and for almost all $x \in X$,

$$
\int_{X}|a(x, y) f(y)| d \mu(y)<\infty \text { and }(A f)(x)=\int_{X} a(x, y) f(y) d \mu(y) .
$$

One can check that a kernel operator $A$ is positive iff its kernel $a$ is non-negative almost everywhere. It is well-known that kernel operators play a very important, often even central, role in a variety of applications from differential and integro-differential equations, problems from physics (in particular from thermodynamics), engineering, statistical and economic models, etc. (see e.g. [16], [4], [8], [17] and the references cited there). For the theory of Banach function spaces 
and more general Banach lattices we refer the reader to the books [24], [5], [1], $[2]$.

Let $A$ and $B$ be positive kernel operators on $L$ with kernels $a$ and $b$ respectively, and $\alpha \geq 0$. The Hadamard (or Schur) product $A \circ B$ of $A$ and $B$ is the kernel operator with kernel equal to $a(x, y) b(x, y)$ at a point $(x, y) \in X \times X$ which can be defined (in general) only on some order ideal of $L$. Similarly, the Hadamard (or Schur) power $A^{(\alpha)}$ of $A$ is the kernel operator with kernel equal to $(a(x, y))^{\alpha}$ at point $(x, y) \in X \times X$ which can be defined only on some order ideal of $L$. Here we use the convention $0^{0}=1$.

Let $A_{1}, \ldots, A_{n}$ be positive kernel operators on a Banach function space $L$, and $\alpha_{1}, \ldots, \alpha_{n}$ positive numbers such that $\sum_{j=1}^{n} \alpha_{j}=1$. Then the Hadamard weighted geometric mean $A=A_{1}^{\left(\alpha_{1}\right)} \circ A_{2}^{\left(\alpha_{2}\right)} \circ \cdots \circ A_{n}^{\left(\alpha_{n}\right)}$ of the operators $A_{1}, \ldots, A_{n}$ is a positive kernel operator defined on the whole space $L$, since $A \leq \alpha_{1} A_{1}+\alpha_{2} A_{2}+$ $\ldots+\alpha_{n} A_{n}$ by the inequality between the weighted arithmetic and geometric means. Let us recall the following result which was proved in [9, Theorem 2.2] and [18, Theorem 5.1].

Theorem 2.1. Let $\left\{A_{i j}\right\}_{i=1, j=1}^{k, m}$ be positive kernel operators on a Banach function space $L$. If $\alpha_{1}, \alpha_{2}, \ldots, \alpha_{m}$ are positive numbers such that $\sum_{j=1}^{m} \alpha_{j}=1$, then the positive kernel operator

$$
A:=\left(A_{11}^{\left(\alpha_{1}\right)} \circ \cdots \circ A_{1 m}^{\left(\alpha_{m}\right)}\right) \ldots\left(A_{k 1}^{\left(\alpha_{1}\right)} \circ \cdots \circ A_{k m}^{\left(\alpha_{m}\right)}\right)
$$

satisfies the following inequalities

$$
\begin{aligned}
A & \leq\left(A_{11} \cdots A_{k 1}\right)^{\left(\alpha_{1}\right)} \circ \cdots \circ\left(A_{1 m} \cdots A_{k m}\right)^{\left(\alpha_{m}\right)} \\
\|A\| & \leq\left\|A_{11} \cdots A_{k 1}\right\|^{\alpha_{1}} \cdots\left\|A_{1 m} \cdots A_{k m}\right\|^{\alpha_{m}} \\
\rho(A) & \leq \rho\left(A_{11} \cdots A_{k 1}\right)^{\alpha_{1}} \cdots \rho\left(A_{1 m} \cdots A_{k m}\right)^{\alpha_{m}} .
\end{aligned}
$$

If, in addition, $L=L^{2}(X, \mu)$, then

$$
w(A) \leq w\left(A_{11} \cdots A_{k 1}\right)^{\alpha_{1}} \cdots w\left(A_{1 m} \cdots A_{k m}\right)^{\alpha_{m}}
$$

The following result is a special case of Theorem 2.1.

Theorem 2.2. Let $A_{1}, \ldots, A_{m}$ be positive kernel operators on a Banach function space $L$, and $\alpha_{1}, \ldots, \alpha_{m}$ positive numbers such that $\sum_{j=1}^{m} \alpha_{j}=1$. Then we have

$$
\left\|A_{1}^{\left(\alpha_{1}\right)} \circ A_{2}^{\left(\alpha_{2}\right)} \circ \cdots \circ A_{m}^{\left(\alpha_{m}\right)}\right\| \leq\left\|A_{1}\right\|^{\alpha_{1}}\left\|A_{2}\right\|^{\alpha_{2}} \cdots\left\|A_{m}\right\|^{\alpha_{m}}
$$

and

$$
\rho\left(A_{1}^{\left(\alpha_{1}\right)} \circ A_{2}^{\left(\alpha_{2}\right)} \circ \cdots \circ A_{m}^{\left(\alpha_{m}\right)}\right) \leq \rho\left(A_{1}\right)^{\alpha_{1}} \rho\left(A_{2}\right)^{\alpha_{2}} \cdots \rho\left(A_{m}\right)^{\alpha_{m}} .
$$

If, in addition, $L=L^{2}(X, \mu)$, then

$$
w\left(A_{1}^{\left(\alpha_{1}\right)} \circ A_{2}^{\left(\alpha_{2}\right)} \circ \cdots \circ A_{m}^{\left(\alpha_{m}\right)}\right) \leq w\left(A_{1}\right)^{\alpha_{1}} w\left(A_{2}\right)^{\alpha_{2}} \cdots w\left(A_{m}\right)^{\alpha_{m}} .
$$

The following refinement of Theorem 2.1, which will be applied in Sections 3 and 4, follows directly from (2.2) and Theorem 2.2. 
Theorem 2.3. Let $L,\left\{A_{i j}\right\}_{i=1, j=1}^{k, m}, \alpha_{1}, \alpha_{2}, \ldots, \alpha_{m}$ and $A$ be as in Theorem 2.1. Then we have

$$
\begin{aligned}
\|A\| & \leq\left\|\left(A_{11} \cdots A_{k 1}\right)^{\left(\alpha_{1}\right)} \circ \cdots \circ\left(A_{1 m} \cdots A_{k m}\right)^{\left(\alpha_{m}\right)}\right\| \\
& \leq\left\|A_{11} \cdots A_{k 1}\right\|^{\alpha_{1}} \cdots\left\|A_{1 m} \cdots A_{k m}\right\|^{\alpha_{m}} \\
\rho(A) & \leq \rho\left(\left(A_{11} \cdots A_{k 1}\right)^{\left(\alpha_{1}\right)} \circ \cdots \circ\left(A_{1 m} \cdots A_{k m}\right)^{\left(\alpha_{m}\right)}\right) \\
& \leq \rho\left(A_{11} \cdots A_{k 1}\right)^{\alpha_{1}} \cdots \rho\left(A_{1 m} \cdots A_{k m}\right)^{\alpha_{m}} .
\end{aligned}
$$

If, in addition, $L=L^{2}(X, \mu)$, then

$$
\begin{aligned}
w(A) & \leq w\left(\left(A_{11} \cdots A_{k 1}\right)^{\left(\alpha_{1}\right)} \circ \cdots \circ\left(A_{1 m} \cdots A_{k m}\right)^{\left(\alpha_{m}\right)}\right) \\
& \leq w\left(A_{11} \cdots A_{k 1}\right)^{\alpha_{1}} \cdots w\left(A_{1 m} \cdots A_{k m}\right)^{\alpha_{m}}
\end{aligned}
$$

The above results on the spectral radius and operator norm remain valid under less restrictive assumption $\sum_{j=1}^{m} \alpha_{j} \geq 1$ in the case of (finite or infinite) nonnegative matrices that define operators on Banach sequence spaces. To make this precise we fix some notations.

Let $R$ denote either the set $\{1, \ldots, n\}$ for some $n \in \mathbb{N}$ or the set $\mathbb{N}$ of all natural numbers. Let $S(R)$ be the vector lattice of all complex sequences $\left(x_{i}\right)_{i \in R}$. A Banach space $L \subseteq S(R)$ is called a Banach sequence space if $x \in S(R), y \in L$ and $|x| \leq|y|$ imply that $x \in L$ and $\|x\|_{L} \leq\|y\|_{L}$.

Let us denote by $\mathcal{L}$ the collection of all Banach sequence spaces $L$ satisfying the property that $e_{i}=\chi_{\{i\}} \in L$ and $\left\|e_{i}\right\|_{L}=1$ for all $i \in R$. Observe that a Banach sequence space is a Banach function space over a measure space $(R, \mu)$, where $\mu$ denotes the counting measure on $R$ (and for $L \in \mathcal{L}$ the set $R$ is the carrier of $L$ ). Standard examples of spaces from $\mathcal{L}$ are Euclidean spaces, the well-known spaces $l^{p}(R)(1 \leq p \leq \infty)$ and the space $c_{0}$ of all null convergent sequences, equipped with the usual norms. The set $\mathcal{L}$ also contains all cartesian products $L=E \times F$ for $E, F \in \mathcal{L}$.

A matrix $A=\left[a_{i j}\right]_{i, j \in R}$ is called non-negative if $a_{i j} \geq 0$ for all $i, j \in R$.

The following result follows from [9, Theorem 3.3] and [18, Theorem 5.1 and Remark 5.2].

Theorem 2.4. Given $L \in \mathcal{L}$, let $\left\{A_{i j}\right\}_{i=1, j=1}^{k, m}$ be non-negative matrices that define operators on $L$. If $\alpha_{1}, \alpha_{2}, \ldots, \alpha_{m}$ are positive numbers such that $\sum_{j=1}^{m} \alpha_{j} \geq 1$, then the matrix $A:=\left(A_{11}^{\left(\alpha_{1}\right)} \circ \cdots \circ A_{1 m}^{\left(\alpha_{m}\right)}\right) \cdots\left(A_{k 1}^{\left(\alpha_{1}\right)} \circ \cdots \circ A_{k m}^{\left(\alpha_{m}\right)}\right)$ also defines an operator on $L$ and it satisfies the inequalities (2.2), (2.9) and (2.10).

Corollary 2.5. Given $L \in \mathcal{L}$, let $A_{1}, \ldots, A_{m}$ be non-negative matrices that define operators on $L$ and $\alpha_{1}, \alpha_{2}, \ldots, \alpha_{m}$ positive numbers such that $\sum_{i=1}^{m} \alpha_{i} \geq 1$. Then the matrix $A_{1}^{\left(\alpha_{1}\right)} \circ A_{2}^{\left(\alpha_{2}\right)} \circ \cdots \circ A_{m}^{\left(\alpha_{m}\right)}$ defines a positive operator on $L$ and the inequalities (2.6) and (2.7) hold.

Note that Theorems 2.1, 2.3 and 2.4 and its special cases proved to be quite useful in different contexts (see e.g. [12], [13], [9], [18], [19], [22], [10], [20], [6]), 
[11], [21] and the references cited there). They will also be some of the main tools in the current article.

We will frequently use the equality $\rho(A B)=\rho(B A)$ that holds for all bounded operators $A$ and $B$ on a Banach space.

\section{Results For POSitive KeRnel operators on BANACH FUnCTION SPACES}

Let $\alpha_{1}, \alpha_{2}, \ldots, \alpha_{m}$ be positive numbers such that $\sum_{j=1}^{m} \alpha_{j}=1$ and let $A_{1}, \ldots, A_{m}$ be positive kernel operators on a Banach function space $L$. We define positive kernel operators $B_{1}, \ldots, B_{m}$ on $L$ in the following way

$$
\begin{aligned}
B_{1} & =A_{1}^{\left(\alpha_{1}\right)} \circ A_{2}^{\left(\alpha_{2}\right)} \circ \cdots \circ A_{m}^{\left(\alpha_{m}\right)}, \\
B_{2} & =A_{2}^{\left(\alpha_{1}\right)} \circ A_{3}^{\left(\alpha_{2}\right)} \circ \cdots \circ A_{1}^{\left(\alpha_{m}\right)}, \\
& \cdots \\
B_{m} & =A_{m}^{\left(\alpha_{1}\right)} \circ A_{1}^{\left(\alpha_{2}\right)} \circ \cdots \circ A_{m-1}^{\left(\alpha_{m}\right)} .
\end{aligned}
$$

In short,

$$
B_{i}=A_{i}^{\left(\alpha_{1}\right)} \circ A_{i+1}^{\left(\alpha_{2}\right)} \circ \cdots \circ A_{m}^{\left(\alpha_{m-i+1}\right)} \circ A_{1}^{\left(\alpha_{m-i+2}\right)} \circ \cdots \circ A_{i-1}^{\left(\alpha_{m}\right)}
$$

for $i=1, \ldots, m$.

First we generalize inequality (1.5) by applying (2.4).

Theorem 3.1. Let $A_{1}, \ldots, A_{m}$ be positive kernel operators on a Banach function space $L$ and let $\alpha_{1}, \alpha_{2}, \ldots, \alpha_{m}$ be positive numbers such that $\sum_{j=1}^{m} \alpha_{j}=1$. If $B_{1}, \ldots, B_{m}$ are operators defined in (3.1), then

$$
\rho\left(B_{1} B_{2} \cdots B_{m}\right) \leq \rho\left(A_{1} A_{2} \cdots A_{m}\right) .
$$

Proof. By (2.4) we have

$$
\begin{gathered}
\rho\left(B_{1} \cdots B_{m}\right) \leq \rho\left(A_{1} A_{2} \ldots A_{m}\right)^{\alpha_{1}} \rho\left(A_{2} \ldots A_{m} A_{1}\right)^{\alpha_{2}} \cdots \rho\left(A_{m} A_{1} \ldots A_{m-1}\right)^{\alpha_{m}} \\
=\rho\left(A_{1} \cdots A_{m}\right)
\end{gathered}
$$

since $\sum_{j=1}^{m} \alpha_{j}=1$ and this completes the proof.

In fact, if we apply in the proof above instead of (2.4) the inequalities (2.10), (2.9), (2.11), we obtain the following refinement of (3.2) and its versions for the operator norm and numerical radius.

Theorem 3.2. Let $L, A_{j}, B_{j}$ and $\alpha_{j}, j=1, \ldots, m$ be as in Theorem 3.1 and let $P_{j}=A_{j} \ldots A_{m} A_{1} \ldots A_{j-1}$ for $j=1, \ldots, m$. Then

$$
\begin{aligned}
& \rho\left(B_{1} B_{2} \cdots B_{m}\right) \leq \rho\left(P_{1}^{\left(\alpha_{1}\right)} \circ P_{2}^{\left(\alpha_{2}\right)} \circ \cdots \circ P_{m}^{\left(\alpha_{m}\right)}\right) \leq \rho\left(A_{1} A_{2} \cdots A_{m}\right), \\
& \left\|B_{1} B_{2} \cdots B_{m}\right\| \leq\left\|P_{1}^{\left(\alpha_{1}\right)} \circ P_{2}^{\left(\alpha_{2}\right)} \circ \cdots \circ P_{m}^{\left(\alpha_{m}\right)}\right\| \leq\left\|P_{1}\right\|^{\alpha_{1}}\left\|P_{2}\right\|^{\alpha_{2}} \cdots\left\|P_{m}\right\|^{\alpha_{m}} . \\
& \text { If, in addition, } L=L^{2}(X, \mu) \text {, then }
\end{aligned}
$$

$w\left(B_{1} B_{2} \cdots B_{m}\right) \leq w\left(P_{1}^{\left(\alpha_{1}\right)} \circ P_{2}^{\left(\alpha_{2}\right)} \circ \cdots \circ P_{m}^{\left(\alpha_{m}\right)}\right) \leq w\left(P_{1}\right)^{\alpha_{1}} w\left(P_{2}\right)^{\alpha_{2}} \cdots w\left(P_{m}\right)^{\alpha_{m}}$. 
In the special case $\alpha_{i}=\frac{1}{m}$ for $i=1, \ldots, m$, we obtain the following refinement of (1.5) and its operator norm and numerical radius versions.

Corollary 3.3. Let $A_{1}, \ldots, A_{m}$ be positive kernel operators on a Banach function space $L$ and let $P_{j}=A_{j} \ldots A_{m} A_{1} \ldots A_{j-1}$ for $j=1, \ldots, m$. Then

$$
\begin{gathered}
\rho\left(A_{1}^{\left(\frac{1}{m}\right)} \circ A_{2}^{\left(\frac{1}{m}\right)} \circ \cdots \circ A_{m}^{\left(\frac{1}{m}\right)}\right) \\
\leq \rho\left(P_{1}^{\left(\frac{1}{m}\right)} \circ P_{2}^{\left(\frac{1}{m}\right)} \circ \cdots \circ P_{m}^{\left(\frac{1}{m}\right)}\right)^{\frac{1}{m}} \leq \rho\left(A_{1} A_{2} \cdots A_{m}\right)^{\frac{1}{m}} . \\
\left\|\left(A_{1}^{\left(\frac{1}{m}\right)} \circ A_{2}^{\left(\frac{1}{m}\right)} \circ \cdots \circ A_{m}^{\left(\frac{1}{m}\right)}\right)^{m}\right\| \\
\leq\left\|P_{1}^{\left(\frac{1}{m}\right)} \circ P_{2}^{\left(\frac{1}{m}\right)} \circ \cdots \circ P_{m}^{\left(\frac{1}{m}\right)}\right\| \leq\left\|P_{1}\right\|^{\frac{1}{m}}\left\|P_{2}\right\|^{\frac{1}{m}} \cdots\left\|P_{m}\right\|^{\frac{1}{m}} .
\end{gathered}
$$

If, in addition, $L=L^{2}(X, \mu)$, then

$$
\begin{gathered}
w\left(\left(A_{1}^{\left(\frac{1}{m}\right)} \circ A_{2}^{\left(\frac{1}{m}\right)} \circ \cdots \circ A_{m}^{\left(\frac{1}{m}\right)}\right)^{m}\right) \\
\leq w\left(P_{1}^{\left(\frac{1}{m}\right)} \circ P_{2}^{\left(\frac{1}{m}\right)} \circ \cdots \circ P_{m}^{\left(\frac{1}{m}\right)}\right) \leq w\left(P_{1}\right)^{\frac{1}{m}} w\left(P_{2}\right)^{\frac{1}{m}} \cdots w\left(P_{m}\right)^{\frac{1}{m}} .
\end{gathered}
$$

Proof. Since $\alpha_{i}=\frac{1}{m}$ for $i=1, \ldots, m$, we have

$$
B_{1}=B_{2}=\cdots=B_{m}=A_{1}^{\left(\frac{1}{m}\right)} \circ A_{2}^{\left(\frac{1}{m}\right)} \circ \cdots \circ A_{m}^{\left(\frac{1}{m}\right)}
$$

and thus (3.3) implies (3.6). Similarly the inequalities (3.7) and (3.8) follow from (3.4) and (3.5), respectively.

Remark 3.4. The inequalities (3.6) could also be deduced from the proof of [21, Theorem 3.4].

The following special case of Theorem 3.2 and Corollary 3.3 refines and generalizes [22, Theorem 2.8] for the spectral radius and points out its operator norm and numerical radius versions.

Corollary 3.5. Let $A$ and $B$ be positive kernel operators on a Banach function space $L$ and let $\alpha \in[0,1]$. Then the following inequalities hold

$$
\begin{gathered}
\rho\left(\left(A^{(\alpha)} \circ B^{(1-\alpha)}\right)\left(B^{(\alpha)} \circ A^{(1-\alpha)}\right)\right) \leq \rho\left((A B)^{(\alpha)} \circ(B A)^{(1-\alpha)}\right) \leq \rho(A B), \\
\rho\left(A^{\left(\frac{1}{2}\right)} \circ B^{\left(\frac{1}{2}\right)}\right) \leq \rho\left((A B)^{\left(\frac{1}{2}\right)} \circ(B A)^{\left(\frac{1}{2}\right)}\right)^{\frac{1}{2}} \leq \rho(A B)^{\frac{1}{2}}, \\
\left\|\left(A^{(\alpha)} \circ B^{(1-\alpha)}\right)\left(B^{(\alpha)} \circ A^{(1-\alpha)}\right)\right\| \leq\left\|(A B)^{(\alpha)} \circ(B A)^{(1-\alpha)}\right\| \leq\|A B\|^{\alpha}\|B A\|^{1-\alpha}, \\
\left\|\left(A^{\left(\frac{1}{2}\right)} \circ B^{\left(\frac{1}{2}\right)}\right)^{2}\right\| \leq\left\|(A B)^{\left(\frac{1}{2}\right)} \circ(B A)^{\left(\frac{1}{2}\right)}\right\| \leq\|A B\|^{\frac{1}{2}}\|B A\|^{\frac{1}{2}} .
\end{gathered}
$$

If, in addition, $L=L^{2}(X, \mu)$, then $w\left(\left(A^{(\alpha)} \circ B^{(1-\alpha)}\right)\left(B^{(\alpha)} \circ A^{(1-\alpha)}\right)\right) \leq w\left((A B)^{(\alpha)} \circ(B A)^{(1-\alpha)}\right) \leq w(A B)^{\alpha} w(B A)^{1-\alpha}$, 


$$
w\left(\left(A^{\left(\frac{1}{2}\right)} \circ B^{\left(\frac{1}{2}\right)}\right)^{2}\right) \leq w\left((A B)^{\left(\frac{1}{2}\right)} \circ(B A)^{\left(\frac{1}{2}\right)}\right) \leq w(A B)^{\frac{1}{2}} w(B A)^{\frac{1}{2}} .
$$

In the case of non-negative matrices that define operators on sequence spaces Theorem 2.4 yields additional results in the case $\sum_{i=1}^{m} \alpha_{i} \geq 1$. More precisely, by applying Theorem 2.4 instead of Theorem 2.3 in the proof of Theorem 3.2, we obtain the following result.

Theorem 3.6. Given $L \in \mathcal{L}$, let $A_{1}, \ldots, A_{m}$ be non-negative matrices that define operators on $L$ and $\alpha_{1}, \alpha_{2}, \ldots, \alpha_{m}$ positive numbers such that $\alpha:=\sum_{i=1}^{m} \alpha_{i} \geq 1$. If $B_{1}, \ldots, B_{m}$ are the operators defined by (3.1) and $P_{j}=A_{j} \ldots A_{m} A_{1} \ldots A_{j-1}$ for $j=1, \ldots, m$, then the inequalities

$$
\rho\left(B_{1} B_{2} \cdots B_{m}\right) \leq \rho\left(P_{1}^{\left(\alpha_{1}\right)} \circ P_{2}^{\left(\alpha_{2}\right)} \circ \cdots \circ P_{m}^{\left(\alpha_{m}\right)}\right) \leq \rho\left(A_{1} A_{2} \cdots A_{m}\right)^{\alpha},
$$

and (3.4) hold.

If, in addition, $L=l^{2}(R)$, then

$$
w\left(B_{1} B_{2} \cdots B_{m}\right) \leq w\left(P_{1}^{\left(\alpha_{1}\right)} \circ P_{2}^{\left(\alpha_{2}\right)} \circ \cdots \circ P_{m}^{\left(\alpha_{m}\right)}\right) .
$$

Remark 3.7. (i) In the special case case $\alpha_{i}=1$ for all $i=1, \ldots, m$ the inequality (3.9) reduces to

$$
\rho\left(A_{1} \circ A_{2} \circ \cdots \circ A_{m}\right) \leq \rho\left(P_{1} \circ P_{2} \circ \cdots \circ P_{m}\right)^{1 / m} \leq \rho\left(A_{1} A_{2} \cdots A_{m}\right) .
$$

Further refinements of (3.11) and of its operator norm and numerical radius counterparts were obtained in [11, Theorem 3.2].

(ii) It follows from the example in [11, Remark 3.8], that in the case $\sum_{i=1}^{m} \alpha_{i} \geq$ 1, the analogue of (3.4) for the numerical radius does not hold.

\section{Further RESUlts ON $L^{2}(X, \mu)$}

In this section we obtain additional inequalities for positive kernel operators on $L^{2}(X, \mu)$, which generalize several results from [11], [15], [6] that were proved there for (finite or infinite) non-negative matrices that define operators on sequence spaces.

If $A$ is a positive kernel operator on $L^{2}(X, \mu)$ with a kernel $a(x, y)$, then its (Hilbert space) adjoint $A^{*}$ is also a positive kernel operator on $L^{2}(X, \mu)$ with a kernel $a(y, x)$. So the operator $S=A^{*} A$ is again a positive kernel operator on $L^{2}(X, \mu)$ with a kernel $s(x, y)=\int_{X} a(z, x) a(z, y) d \mu(z)$ and $S$ is also a positive semidefinite operator. In what follows we will use the following well-known equalities

$$
\|A\|^{2}=\left\|A A^{*}\right\|=\left\|A^{*} A\right\|=\rho\left(A A^{*}\right)=\rho\left(A^{*} A\right) .
$$

The inequalities (4.2) bellow are the kernel version of a matrix result [11, Corollary 3.5] and they also generalize and refine [6, Proposition 2.4] and [15, Theorem 4], while the inequalities (4.3) refine $(2.6)$ in the $L^{2}(X, \mu)$ case. 
Theorem 4.1. Let $A_{1}, \ldots, A_{m}$ be positive kernel operators on $L^{2}(X, \mu)$ and let $S_{j}=A_{j}^{*} A_{j}, Q_{j}=S_{j} \ldots S_{m} S_{1} \ldots S_{j-1}$ for $j=1, \ldots, m$. Assume that $\alpha_{1}, \alpha_{2}, \ldots$, $\alpha_{m}$ are positive numbers such that $\sum_{j=1}^{m} \alpha_{j}=1$. Then we have

$$
\begin{gathered}
\left\|A_{1}^{\left(\frac{1}{m}\right)} \circ A_{2}^{\left(\frac{1}{m}\right)} \circ \cdots \circ A_{m}^{\left(\frac{1}{m}\right)}\right\| \leq \rho\left(S_{1}^{\left(\frac{1}{m}\right)} \circ S_{2}^{\left(\frac{1}{m}\right)} \circ \cdots \circ S_{m}^{\left(\frac{1}{m}\right)}\right)^{\frac{1}{2}} \\
\quad \leq \rho\left(Q_{1}^{\left(\frac{1}{m}\right)} \circ Q_{2}^{\left(\frac{1}{m}\right)} \circ \cdots \circ Q_{m}^{\left(\frac{1}{m}\right)}\right)^{\frac{1}{2 m}} \leq \rho\left(S_{1} S_{2} \cdots S_{m}\right)^{\frac{1}{2 m}}
\end{gathered}
$$

and

$$
\left\|A_{1}^{\left(\alpha_{1}\right)} \circ \cdots \circ A_{m}^{\left(\alpha_{m}\right)}\right\| \leq \rho\left(S_{1}^{\left(\alpha_{1}\right)} \circ \cdots \circ S_{m}^{\left(\alpha_{m}\right)}\right)^{\frac{1}{2}} \leq\left\|A_{1}\right\|^{\alpha_{1}} \cdots\left\|A_{m}\right\|^{\alpha_{m}}
$$

Proof. First observe that

$$
\begin{gathered}
\left(A_{1}^{\left(\alpha_{1}\right)} \circ \cdots \circ A_{m}^{\left(\alpha_{m}\right)}\right)^{*}\left(A_{1}^{\left(\alpha_{1}\right)} \circ \cdots \circ A_{m}^{\left(\alpha_{m}\right)}\right) \\
=\left(\left(A_{1}^{*}\right)^{\left(\alpha_{1}\right)} \circ \cdots \circ\left(A_{m}^{*}\right)^{\left(\alpha_{m}\right)}\right)\left(A_{1}^{\left(\alpha_{1}\right)} \circ \cdots \circ A_{m}^{\left(\alpha_{m}\right)}\right) \leq\left(A_{1}^{*} A_{1}\right)^{\left(\alpha_{1}\right)} \circ \cdots \circ\left(A_{m}^{*} A_{m}\right)^{\left(\alpha_{m}\right)} \\
=S_{1}^{\left(\alpha_{1}\right)} \circ S_{2}^{\left(\alpha_{2}\right)} \circ \cdots \circ S_{m}^{\left(\alpha_{m}\right)}
\end{gathered}
$$

by (2.2). The first inequalities in (4.2) and (4.3) follow from (4.4), the monotonicity of spectral radius and (4.1). The remaining inequalities in (4.2) follow from (3.6).

The remaining inequalities in (4.3) follow from (2.7) and (4.1):

$$
\begin{aligned}
& \left\|A_{1}^{\left(\alpha_{1}\right)} \circ \cdots \circ A_{m}^{\left(\alpha_{m}\right)}\right\| \leq \rho\left(S_{1}^{\left(\alpha_{1}\right)} \circ \cdots \circ S_{m}^{\left(\alpha_{m}\right)}\right)^{\frac{1}{2}} \\
\leq & \left(\rho\left(S_{1}\right)^{\alpha_{1}} \cdots \rho\left(S_{m}\right)^{\alpha_{m}}\right)^{\frac{1}{2}}=\left\|A_{1}\right\|^{\alpha_{1}} \cdots\left\|A_{m}\right\|^{\alpha_{m}}
\end{aligned}
$$

In the case of non-negative matrices that define operators on $l^{2}(R)$ the following generalization of (4.3) holds.

Theorem 4.2. Let $A_{1}, \ldots, A_{m}$ be non-negative matrices that define operators on $l^{2}(R)$ and let $\alpha_{1}, \alpha_{2}, \ldots, \alpha_{m}$ be positive numbers such that $\sum_{j=1}^{m} \alpha_{j} \geq 1$. If $S_{j}=A_{j}^{T} A_{j}$ for $j=1, \ldots, m$, then (4.3) holds.

Proof. The result follows by applying Theorem 2.4 instead of Theorem 2.3 in the proof of Theorem 4.1.

The following result is a direct consequence of previous two theorems in the case of two operators.

Corollary 4.3. Let $A$ and $B$ be positive kernel operators on $L^{2}(X, \mu)$. Then

$$
\begin{gathered}
\left\|A^{\left(\frac{1}{2}\right)} \circ B^{\left(\frac{1}{2}\right)}\right\| \leq \rho\left(\left(A^{*} A\right)^{\left(\frac{1}{2}\right)} \circ\left(B^{*} B\right)^{\left(\frac{1}{2}\right)}\right)^{\frac{1}{2}} \\
\leq \rho\left(\left(A^{*} A B^{*} B\right)^{\left(\frac{1}{2}\right)} \circ\left(B^{*} B A^{*} A\right)^{\left(\frac{1}{2}\right)}\right)^{\frac{1}{4}} \leq \rho\left(A^{*} A B^{*} B\right)^{\frac{1}{4}}=\left\|A B^{*}\right\|^{\frac{1}{2}} \leq\|A\|^{\frac{1}{2}}\|B\|^{\frac{1}{2}}
\end{gathered}
$$

and

$$
\left\|A^{(\alpha)} \circ B^{(1-\alpha)}\right\| \leq \rho\left(\left(A^{*} A\right)^{(\alpha)} \circ\left(B^{*} B\right)^{(1-\alpha)}\right)^{\frac{1}{2}} \leq\|A\|^{\alpha}\|B\|^{1-\alpha},
$$

if $\alpha \in[0,1]$. 
If, in addition, $L=l^{2}(R)$ (and so $A$ and $B$ may be considered as non-negative matrices that define operators on $\left.l^{2}(R)\right)$, then

$$
\left\|A^{(\alpha)} \circ B^{(\beta)}\right\| \leq \rho\left(\left(A^{*} A\right)^{(\alpha)} \circ\left(B^{*} B\right)^{(\beta)}\right)^{\frac{1}{2}} \leq\|A\|^{\alpha}\|B\|^{\beta},
$$

whenever $\alpha, \beta>0$ such that $\alpha+\beta \geq 1$.

Proof. Inequalities (4.6), (4.7) and the first three inequalities in (4.5) are special cases of Theorems 4.1 and 4.2. To complete the proof (4.5) observe that,

$$
\rho\left(A^{*} A B^{*} B\right)=\rho\left(A B^{*} B A^{*}\right)=\rho\left(A B^{*}\left(A B^{*}\right)^{*}\right)=\left\|A B^{*}\right\|^{2} \leq\|A\|^{2}\|B\|^{2},
$$

where the third equality follows from (4.1).

If $A$ and $B$ are non-negative matrices that define operators on $l^{2}(R)$, then the inequalities (1.6) were proved in [11, Corollary 3.10] and previously in the finite dimensional case in [6, Corollary 2.3] and [15, Corollary 6]. Consequently, we have

$$
\|A \circ B\| \leq \rho^{\frac{1}{2}}\left(\left(A^{T} B\right) \circ\left(B^{T} A\right)\right) \leq \rho\left(A^{T} B\right)=\rho\left(A B^{T}\right) \leq\left\|A B^{T}\right\| \leq\|A\|\|B\| .
$$

The following result proves a version of this result for positive kernel operators and generalizes it even in the case of non-negative matrices that define operators on $l^{2}(R)$.

Theorem 4.4. Let $A$ and $B$ be positive kernel operators on $L^{2}(X, \mu)$. Then

$$
\left\|A^{\left(\frac{1}{2}\right)} \circ B^{\left(\frac{1}{2}\right)}\right\| \leq \rho^{\frac{1}{2}}\left(\left(A^{*} B\right)^{\left(\frac{1}{2}\right)} \circ\left(B^{*} A\right)^{\left(\frac{1}{2}\right)}\right) \leq \rho^{\frac{1}{2}}\left(A^{*} B\right) .
$$

If, in addition, $L=l^{2}(R)$, then

$$
\left\|A^{(\alpha)} \circ B^{(\alpha)}\right\| \leq \rho^{\frac{1}{2}}\left(\left(A^{T} B\right)^{(\alpha)} \circ\left(B^{T} A\right)^{(\alpha)}\right) \leq \rho^{\alpha}\left(A^{T} B\right),
$$

whenever $\alpha \geq \frac{1}{2}$.

Proof. First we prove (4.8). By (4.1) and Theorem 2.3 we have

$$
\begin{gathered}
\left\|A^{\left(\frac{1}{2}\right)} \circ B^{\left(\frac{1}{2}\right)}\right\|^{2}=\rho\left(\left(\left(A^{*}\right)^{\left(\frac{1}{2}\right)} \circ\left(B^{*}\right)^{\left(\frac{1}{2}\right)}\right)\left(B^{\left(\frac{1}{2}\right)} \circ A^{\left(\frac{1}{2}\right)}\right)\right) \leq \rho\left(\left(A^{*} B\right)^{\left(\frac{1}{2}\right)} \circ\left(B^{*} A\right)^{\left(\frac{1}{2}\right)}\right) \\
\leq \rho^{\frac{1}{2}}\left(A^{*} B\right) \rho^{\frac{1}{2}}\left(B^{*} A\right)=\rho\left(A^{*} B\right),
\end{gathered}
$$

where the last equality follows from $\rho\left(B^{*} A\right)=\rho\left(\left(B^{*} A\right)^{*}\right)=\rho\left(A^{*} B\right)$. This proves (4.8).

The inequalities (4.9) are proved in a similar way by applying Theorem 2.4 instead of Theorem 2.3.

Remark 4.5. If $A$ and $B$ are positive kernel operators on $L^{2}(X, \mu)$, then (4.8) implies

$$
\begin{gathered}
\left\|A^{\left(\frac{1}{2}\right)} \circ B^{\left(\frac{1}{2}\right)}\right\| \leq \rho^{\frac{1}{2}}\left(\left(A^{*} B\right)^{\left(\frac{1}{2}\right)} \circ\left(B^{*} A\right)^{\left(\frac{1}{2}\right)}\right) \leq \rho^{\frac{1}{2}}\left(A^{*} B\right)=\rho^{\frac{1}{2}}\left(A B^{*}\right) \\
\leq\left\|A B^{*}\right\|^{\frac{1}{2}} \leq\|A\|^{\frac{1}{2}}\|B\|^{\frac{1}{2}}
\end{gathered}
$$

which counterparts the inequalities (4.5). 
The following two theorems generalize Theorem 4.4 to several operators. Theorem 4.6 bellow is a version of [11, Theorem 3.9] and [15, Theorem 5] for kernel operators, while Theorem 4.8 generalizes these results even in the case of nonnegative matrices.

Theorem 4.6. Let $A_{1}, \ldots, A_{m}$ be positive kernel operators on $L^{2}(X, \mu)$.

If $m$ is even, then

$$
\begin{gathered}
\left\|A_{1}^{\left(\frac{1}{m}\right)} \circ A_{2}^{\left(\frac{1}{m}\right)} \circ \cdots \circ A_{m}^{\left(\frac{1}{m}\right)}\right\| \leq\left(\rho\left(A_{1}^{*} A_{2} A_{3}^{*} A_{4} \cdots A_{m-1}^{*} A_{m}\right) \rho\left(A_{1} A_{2}^{*} A_{3} A_{4}^{*} \cdots A_{m-1} A_{m}^{*}\right)\right)^{\frac{1}{2 m}} \\
=\left(\rho\left(A_{1}^{*} A_{2} A_{3}^{*} A_{4} \cdots A_{m-1}^{*} A_{m}\right) \rho\left(A_{m} A_{m-1}^{*} \cdots A_{4} A_{3}^{*} A_{2} A_{1}^{*}\right)\right)^{\frac{1}{2 m}} .
\end{gathered}
$$

If $m$ is odd, then

$$
\begin{gathered}
\left\|A_{1}^{\left(\frac{1}{m}\right)} \circ A_{2}^{\left(\frac{1}{m}\right)} \circ \cdots \circ A_{m}^{\left(\frac{1}{m}\right)}\right\| \\
\leq \rho\left(A_{1} A_{2}^{*} A_{3} A_{4}^{*} \cdots A_{m-2} A_{m-1}^{*} A_{m} A_{1}^{*} A_{2} A_{3}^{*} A_{4} \cdots A_{m-2}^{*} A_{m-1} A_{m}^{*}\right)^{\frac{1}{2 m}}
\end{gathered}
$$

Proof. If $m$ is even, we have by (2.2)

$$
\begin{gathered}
\left(\left(A_{1}^{\left(\frac{1}{m}\right)} \circ A_{2}^{\left(\frac{1}{m}\right)} \circ \cdots \circ A_{m}^{\left(\frac{1}{m}\right)}\right)^{*}\left(A_{1}^{\left(\frac{1}{m}\right)} \circ A_{2}^{\left(\frac{1}{m}\right)} \circ \cdots \circ A_{m}^{\left(\frac{1}{m}\right)}\right)\right)^{\frac{m}{2}} \\
=\left(\left(A_{1}^{*}\right)^{\left(\frac{1}{m}\right)} \circ\left(A_{2}^{*}\right)\left(\frac{1}{m}\right) \circ \cdots \circ\left(A_{m}^{*}\right)\left(\frac{1}{m}\right)\right)\left(A_{2}^{\left(\frac{1}{m}\right)} \circ \cdots \circ A_{m}^{\left(\frac{1}{m}\right)} \circ A_{1}^{\left(\frac{1}{m}\right)}\right) \\
\left(\left(A_{3}^{*}\right)\left(\frac{1}{m}\right) \circ \cdots \circ\left(A_{m}^{*}\right)\left(\frac{1}{m}\right) \circ\left(A_{1}^{*}\right)^{\left(\frac{1}{m}\right)} \circ\left(A_{2}^{*}\right)\left(\frac{1}{m}\right)\right)\left(A_{4}^{\left(\frac{1}{m}\right)} \circ \cdots \circ A_{m}^{\left(\frac{1}{m}\right)} \circ A_{1}^{\left(\frac{1}{m}\right)} \circ A_{2}^{\left(\frac{1}{m}\right)} \circ A_{3}^{\left(\frac{1}{m}\right)}\right) \\
\cdots\left(\left(A_{m-1}^{*}\right)\left(\frac{1}{m}\right) \circ\left(A_{m}^{*}\right)\left(\frac{1}{m}\right) \circ\left(A_{1}^{*}\right)^{\left(\frac{1}{m}\right)} \circ \cdots \circ\left(A_{m-2}^{*}\right)^{\left(\frac{1}{m}\right)}\right)\left(A_{m}^{\left(\frac{1}{m}\right)} \circ A_{1}^{\left(\frac{1}{m}\right)} \circ \cdots \circ A_{m-1}^{\left(\frac{1}{m}\right)}\right) \\
\leq B:=\left(A_{1}^{*} A_{2} A_{3}^{*} A_{4} \cdots A_{m-1}^{*} A_{m}\right)\left(\frac{1}{m}\right) \circ\left(A_{2}^{*} A_{3} A_{4}^{*} A_{5} \cdots A_{m}^{*} A_{1}\right)^{\left(\frac{1}{m}\right)} \circ \cdots \\
\circ\left(A_{m-1}^{*} A_{m} A_{1}^{*} A_{2} \cdots A_{m-3}^{*} A_{m-2}\right)\left(\frac{1}{m}\right) \circ\left(A_{m}^{*} A_{1} A_{2}^{*} A_{3} \cdots A_{m-2}^{*} A_{m-1}\right)^{\left(\frac{1}{m}\right)} .
\end{gathered}
$$

It follows by (2.4) that

$$
\begin{gathered}
\left\|A_{1}^{\left(\frac{1}{m}\right)} \circ A_{2}^{\left(\frac{1}{m}\right)} \circ \cdots \circ A_{m}^{\left(\frac{1}{m}\right)}\right\|^{m}= \\
\rho\left(\left(A_{1}^{\left(\frac{1}{m}\right)} \circ A_{2}^{\left(\frac{1}{m}\right)} \circ \cdots \circ A_{m}^{\left(\frac{1}{m}\right)}\right)^{*}\left(A_{1}^{\left(\frac{1}{m}\right)} \circ A_{2}^{\left(\frac{1}{m}\right)} \circ \cdots \circ A_{m}^{\left(\frac{1}{m}\right)}\right)\right)^{\frac{m}{2}} \\
\leq \rho(B) \leq \rho\left(A_{1}^{*} A_{2} A_{3}^{*} A_{4} \cdots A_{m-1}^{*} A_{m}\right)^{\frac{1}{m}} \rho\left(A_{2}^{*} A_{3} A_{4}^{*} A_{5} \cdots A_{m}^{*} A_{1}\right)^{\frac{1}{m}} \cdots \\
\cdots \rho\left(A_{m-1}^{*} A_{m} A_{1}^{*} A_{2} \cdots A_{m-3}^{*} A_{m-2}\right)^{\frac{1}{m}} \rho\left(A_{m}^{*} A_{1} A_{2}^{*} A_{3} \cdots A_{m-2}^{*} A_{m-1}\right)^{\frac{1}{m}} \\
=\rho^{\frac{1}{2}}\left(A_{1}^{*} A_{2} A_{3}^{*} A_{4} \cdots A_{m-1}^{*} A_{m}\right) \rho^{\frac{1}{2}}\left(A_{1} A_{2}^{*} A_{3} A_{4}^{*} \cdots A_{m-1} A_{m}^{*}\right),
\end{gathered}
$$

which proves (4.10).

If $m$ is odd, we have by (2.2)

$$
\begin{aligned}
& \left(\left(A_{1}^{\left(\frac{1}{m}\right)} \circ A_{2}^{\left(\frac{1}{m}\right)} \circ \cdots \circ A_{m}^{\left(\frac{1}{m}\right)}\right)^{*}\left(A_{1}^{\left(\frac{1}{m}\right)} \circ A_{2}^{\left(\frac{1}{m}\right)} \circ \cdots \circ A_{m}^{\left(\frac{1}{m}\right)}\right)\right)^{m} \\
= & \left(\left(A_{1}^{*}\right)^{\left(\frac{1}{m}\right)} \circ\left(A_{2}^{*}\right)^{\left(\frac{1}{m}\right)} \circ \cdots \circ\left(A_{m}^{*}\right)^{\left(\frac{1}{m}\right)}\right)\left(A_{2}^{\left(\frac{1}{m}\right)} \circ \cdots \circ A_{m}^{\left(\frac{1}{m}\right)} \circ A_{1}^{\left(\frac{1}{m}\right)}\right)
\end{aligned}
$$




$$
\begin{gathered}
\left(\left(A_{3}^{*}\right)^{\left(\frac{1}{m}\right)} \cdots \cdots\left(A_{m}^{*}\right)\left(\frac{1}{m}\right) \circ\left(A_{1}^{*}\right)\left(\frac{1}{m}\right) \circ\left(A_{2}^{*}\right)\left(\frac{1}{m}\right)\right)\left(A_{4}^{\left(\frac{1}{m}\right)} \circ \cdots \circ A_{m}^{\left(\frac{1}{m}\right)} \circ A_{1}^{\left(\frac{1}{m}\right)} \circ A_{2}^{\left(\frac{1}{m}\right)} \circ A_{3}^{\left(\frac{1}{m}\right)}\right) \\
\cdots\left(A_{m-1}^{\left(\frac{1}{m}\right)} \circ A_{m}^{\left(\frac{1}{m}\right)} \circ A_{1}^{\left(\frac{1}{m}\right)} \circ \cdots \circ A_{m-2}^{\left(\frac{1}{m}\right)}\right)\left(\left(A_{m}^{*}\right)^{\left(\frac{1}{m}\right)} \circ\left(A_{1}^{*}\right)^{\left(\frac{1}{m}\right)} \circ \cdots \circ\left(A_{m-1}^{*}\left(\frac{1}{m}\right)\right)\right. \\
\left(A_{1}^{\left(\frac{1}{m}\right)} \circ A_{2}^{\left(\frac{1}{m}\right)} \circ \cdots \circ A_{m}^{\left(\frac{1}{m}\right)}\right)\left(\left(A_{2}^{*}\right)^{\left(\frac{1}{m}\right)} \circ \cdots \circ\left(A_{m}^{*}\right)^{\left(\frac{1}{m}\right)} \circ\left(A_{1}^{*}\right)^{\left(\frac{1}{m}\right)}\right) \\
\left(A_{3}^{\left(\frac{1}{m}\right)} \circ A_{4}^{\left(\frac{1}{m}\right)} \circ \cdots \circ A_{m}^{\left(\frac{1}{m}\right)} \circ A_{1}^{\left(\frac{1}{m}\right)} \circ A_{2}^{\left(\frac{1}{m}\right)}\right) \cdots \\
\ldots\left(\left(A_{m-1}^{*}\right)\left(\frac{1}{m}\right) \circ\left(A_{m}^{*}\right)\left(\frac{1}{m}\right) \circ\left(A_{1}^{*}\right)\left(\frac{1}{m}\right) \circ \cdots \circ\left(A_{m-2}^{*}\right)^{\left(\frac{1}{m}\right)}\right)\left(A_{m}^{\left(\frac{1}{m}\right)} \circ A_{1}^{\left(\frac{1}{m}\right)} \circ \cdots \circ A_{m-1}^{\left(\frac{1}{m}\right)}\right) \\
\leq C:=\left(A_{1}^{*} A_{2} A_{3}^{*} A_{4} \cdots A_{m-1} A_{m}^{*} A_{1} A_{2}^{*} A_{3} A_{4}^{*} \cdots A_{m-1}^{*} A_{m}\right)^{\left(\frac{1}{m}\right)} \circ \\
\left(A_{2}^{*} A_{3} A_{4}^{*} \cdots A_{m-1}^{*} A_{m} A_{1}^{*} A_{2} A_{3}^{*} A_{4} \cdots A_{m-1} A_{m}^{*} A_{1}\right)^{\left(\frac{1}{m}\right)} \circ \cdots \\
\ldots \circ\left(A_{m}^{*} A_{1} A_{2}^{*} A_{3} A_{4}^{*} \cdots A_{m-1}^{*} A_{m} A_{1}^{*} A_{2} A_{3}^{*} A_{4} \cdots A_{m-1}\right)^{\left(\frac{1}{m}\right)} .
\end{gathered}
$$

It follows by (2.4) that

$$
\begin{gathered}
\left\|A_{1}^{\left(\frac{1}{m}\right)} \circ A_{2}^{\left(\frac{1}{m}\right)} \circ \cdots \circ A_{m}^{\left(\frac{1}{m}\right)}\right\|^{2 m} \\
\leq \rho(C) \leq \rho^{\frac{m+1}{2 m}}\left(A_{1}^{*} A_{2} A_{3}^{*} A_{4} \cdots A_{m-1} A_{m}^{*} A_{1} A_{2}^{*} A_{3} A_{4}^{*} \cdots A_{m-1}^{*} A_{m}\right) \times \\
\rho^{\frac{m-1}{2 m}}\left(A_{1} A_{2}^{*} A_{3} A_{4}^{*} \cdots A_{m-1}^{*} A_{m} A_{1}^{*} A_{2} A_{3}^{*} A_{4} \cdots A_{m-1} A_{m}^{*}\right) \\
=\rho\left(A_{1} A_{2}^{*} A_{3} A_{4}^{*} \cdots A_{m-1}^{*} A_{m} A_{1}^{*} A_{2} A_{3}^{*} A_{4} \cdots A_{m-1} A_{m}^{*}\right),
\end{gathered}
$$

which completes the proof.

Remark 4.7. If $A_{1}, \ldots, A_{m}$ are positive kernel operators on $L^{2}(X, \mu)$, then the proof above yields the following refinements of (4.10) and (4.11).

If $m$ is even, then

$$
\begin{gathered}
\left\|A_{1}^{\left(\frac{1}{m}\right)} \circ A_{2}^{\left(\frac{1}{m}\right)} \circ \cdots \circ A_{m}^{\left(\frac{1}{m}\right)}\right\| \leq \rho^{\frac{1}{m}}(B) \\
=\left(\rho\left(A_{1}^{*} A_{2} A_{3}^{*} A_{4} \cdots A_{m-1}^{*} A_{m}\right) \rho\left(A_{m} A_{m-1}^{*} \cdots A_{4} A_{3}^{*} A_{2} A_{1}^{*}\right)\right)^{\frac{1}{2 m}}
\end{gathered}
$$

and, if $m$ is odd, then

$$
\begin{gathered}
\left\|A_{1}^{\left(\frac{1}{m}\right)} \circ A_{2}^{\left(\frac{1}{m}\right)} \circ \cdots \circ A_{m}^{\left(\frac{1}{m}\right)}\right\| \leq \rho^{\frac{1}{2 m}}(C) \\
\leq \rho\left(A_{1} A_{2}^{*} A_{3} A_{4}^{*} \cdots A_{m-2} A_{m-1}^{*} A_{m} A_{1}^{*} A_{2} A_{3}^{*} A_{4} \cdots A_{m-2}^{*} A_{m-1} A_{m}^{*}\right)^{\frac{1}{2 m}},
\end{gathered}
$$

where the operators $B$ and $C$ are defined in (4.12) and (4.13), respectively.

The following theorem generalizes [11, Theorem 3.9] and [15, Theorem 5]. It can be proved in a similar way as Theorem 4.6 by applying Theorem 2.4 instead of Theorem 2.3 in the proof. We omit the details of the proof. 
Theorem 4.8. Let $A_{1}, \ldots, A_{m}$ be non-negative matrices that define operators on $l^{2}(R)$ and let $\alpha \geq \frac{1}{m}$.

If $m$ is even, then

$$
\begin{gathered}
\left\|A_{1}^{(\alpha)} \circ A_{2}^{(\alpha)} \circ \cdots \circ A_{m}^{(\alpha)}\right\| \leq \rho^{\frac{1}{m}}\left(B_{\alpha}\right) \\
\leq\left(\rho\left(A_{1}^{T} A_{2} A_{3}^{T} A_{4} \cdots A_{m-1}^{T} A_{m}\right) \rho\left(A_{m} A_{m-1}^{T} \cdots A_{4} A_{3}^{T} A_{2} A_{1}^{T}\right)\right)^{\frac{\alpha}{2}},
\end{gathered}
$$

where

$$
\begin{aligned}
& B_{\alpha}=\left(A_{1}^{T} A_{2} A_{3}^{T} A_{4} \cdots A_{m-1}^{T} A_{m}\right)^{(\alpha)} \circ\left(A_{2}^{T} A_{3} A_{4}^{T} A_{5} \cdots A_{m}^{T} A_{1}\right)^{(\alpha)} \circ \cdots \\
& \circ\left(A_{m-1}^{T} A_{m} A_{1}^{T} A_{2} \cdots A_{m-3}^{T} A_{m-2}\right)^{(\alpha)} \circ\left(A_{m}^{T} A_{1} A_{2}^{T} A_{3} \cdots A_{m-2}^{T} A_{m-1}\right)^{(\alpha)} .
\end{aligned}
$$

If $m$ is odd, then

$$
\begin{gathered}
\left\|A_{1}^{(\alpha)} \circ A_{2}^{(\alpha)} \circ \cdots \circ A_{m}^{(\alpha)}\right\| \leq \rho^{\frac{1}{2 m}}\left(C_{\alpha}\right) \\
\leq \rho\left(A_{1} A_{2}^{T} A_{3} A_{4}^{T} \cdots A_{m-2} A_{m-1}^{T} A_{m} A_{1}^{T} A_{2} A_{3}^{T} A_{4} \cdots A_{m-2}^{T} A_{m-1} A_{m}^{T}\right)^{\frac{\alpha}{2}},
\end{gathered}
$$

where

$$
\begin{gathered}
C_{\alpha}=\left(A_{1}^{T} A_{2} A_{3}^{T} A_{4} \cdots A_{m-1} A_{m}^{T} A_{1} A_{2}^{T} A_{3} A_{4}^{T} \cdots A_{m-1}^{T} A_{m}\right)^{(\alpha)} \circ \\
\left(A_{2}^{T} A_{3} A_{4}^{T} \cdots A_{m-1}^{T} A_{m} A_{1}^{T} A_{2} A_{3}^{T} A_{4} \cdots A_{m-1} A_{m}^{T} A_{1}\right)^{(\alpha)} \circ \cdots \\
\cdots \circ\left(A_{m}^{T} A_{1} A_{2}^{T} A_{3} A_{4}^{T} \cdots A_{m-1}^{T} A_{m} A_{1}^{T} A_{2} A_{3}^{T} A_{4} \cdots A_{m-1}\right)^{(\alpha)} .
\end{gathered}
$$

The following result, which is a special case of (4.15) and (4.17), generalizes [11, Corollary 3.11].

Corollary 4.9. Let $A_{1}, A_{2}, A_{3}$ be positive kernel operators on $L^{2}(X, \mu)$. Then

$$
\begin{gathered}
\left\|A_{1}^{\left(\frac{1}{3}\right)} \circ A_{2}^{\left(\frac{1}{3}\right)} \circ A_{3}^{\left(\frac{1}{3}\right)}\right\| \leq \\
\rho\left(\left(A_{1}^{*} A_{2} A_{3}^{*} A_{1} A_{2}^{*} A_{3}\right)^{\left(\frac{1}{3}\right)} \circ\left(A_{2}^{*} A_{3} A_{1}^{*} A_{2} A_{3}^{*} A_{1}\right)^{\left(\frac{1}{3}\right)} \circ\left(A_{3}^{*} A_{1} A_{2}^{*} A_{3} A_{1}^{*} A_{2}\right)^{\left(\frac{1}{3}\right)}\right)^{\frac{1}{6}} \\
\leq \rho\left(A_{1} A_{2}^{*} A_{3} A_{1}^{*} A_{2} A_{3}^{*}\right)^{\frac{1}{6}} .
\end{gathered}
$$

If, in addition, $L=l^{2}(R)$ and $\alpha \geq \frac{1}{3}$, then

$$
\begin{gathered}
\left\|A_{1}^{(\alpha)} \circ A_{2}^{(\alpha)} \circ A_{3}^{(\alpha)}\right\| \leq \\
\rho\left(\left(A_{1}^{T} A_{2} A_{3}^{T} A_{1} A_{2}^{T} A_{3}\right)^{(\alpha)} \circ\left(A_{2}^{T} A_{3} A_{1}^{T} A_{2} A_{3}^{T} A_{1}\right)^{(\alpha)} \circ\left(A_{3}^{T} A_{1} A_{2}^{T} A_{3} A_{1}^{T} A_{2}\right)^{(\alpha)}\right)^{\frac{1}{6}} \\
\leq \rho\left(A_{1} A_{2}^{T} A_{3} A_{1}^{T} A_{2} A_{3}^{T}\right)^{\frac{\alpha}{2}} .
\end{gathered}
$$

The following lower bounds for the operator norm of the Jordan triple product $A B A$ generalize [11, Corollary 3.12].

Corollary 4.10. Let $A$ and $B$ be positive kernel operators on $L^{2}(X, \mu)$. Then

$$
\begin{gathered}
\left\|A^{\left(\frac{1}{3}\right)} \circ\left(B^{*}\right)^{\left(\frac{1}{3}\right)} \circ A^{\left(\frac{1}{3}\right)}\right\| \leq \\
\rho\left(\left(A^{*} B^{*} A^{*} A B A\right)^{\left(\frac{1}{3}\right)} \circ\left(B A A^{*} B^{*} A^{*} A\right)^{\left(\frac{1}{3}\right)} \circ\left(A^{*} A B A A^{*} B^{*}\right)\left(\frac{1}{3}\right)\right)^{\frac{1}{6}} \leq\|A B A\|^{\frac{1}{3}} .
\end{gathered}
$$

If, in addition, $L=l^{2}(R)$ and $\alpha \geq \frac{1}{3}$, then

$$
\left\|A^{(\alpha)} \circ\left(B^{T}\right)^{(\alpha)} \circ A^{(\alpha)}\right\| \leq
$$




$$
\rho\left(\left(A^{T} B^{T} A^{T} A B A\right)^{(\alpha)} \circ\left(B A A^{T} B^{T} A^{T} A\right)^{(\alpha)} \circ\left(A^{T} A B A A^{T} B^{T}\right)^{(\alpha)}\right)^{\frac{1}{6}} \leq\|A B A\|^{\alpha} .
$$

Proof. The inequalities (4.20) follow from (4.18) by setting $A_{1}=A_{3}=A$ and $A_{2}=B^{*}$ and observing that

$$
\rho\left(A B A A^{*} B^{*} A^{*}\right)^{\frac{1}{6}}=\rho\left(A B A(A B A)^{*}\right)^{\frac{1}{6}}=\|A B A\|^{\frac{1}{3}} .
$$

Similarly the inequalities (4.21) follow from (4.19).

Remark 4.11. In contrast to the inequalities (4.20) and (4.21) the inequalities $\left\|A^{\left(\frac{1}{3}\right)} \circ B^{\left(\frac{1}{3}\right)} \circ A^{\left(\frac{1}{3}\right)}\right\| \leq\|A B A\|^{\frac{1}{3}}$ and $\left\|A^{(\alpha)} \circ B^{(\alpha)} \circ A^{(\alpha)}\right\| \leq\|A B A\|^{\alpha}$ for $\alpha \geq \frac{1}{3}$ are not valid in general. This is shown by the following example from [15] and [11]. If $A=\left[\begin{array}{ll}0 & 1 \\ 0 & 1\end{array}\right]$ and $B=\left[\begin{array}{ll}1 & 1 \\ 0 & 0\end{array}\right]$, then

$$
\left\|A^{(\alpha)} \circ B^{(\alpha)} \circ A^{(\alpha)}\right\|=\|A \circ B \circ A\|=1>0=\|A B A\|^{\alpha} .
$$

The inequalities (4.21) are sharp, as the case $A=B=I$ shows.

Acknowledgements. The author thanks Professor Franz Lehner for reading the first version of this article and to his collegues and staff at the Institute of Discrete Mathematics of TU Graz for their hospitality during his research stay in Austria.

This work was supported in part by the JESH grant of the Austrian Academy of Sciences and by grant P1-0222 of the Slovenian Research Agency.

\section{REFERENCES}

1. Y.A. Abramovich and C.D. Aliprantis, An invitation to operator theory, American Mathematical Society, Providence, 2002.

2. C.D. Aliprantis and O. Burkinshaw, Positive operators, Springer, Dordrecht, 2006.

3. K.M.R. Audenaert, Spectral radius of Hadamard product versus conventional product for non-negative matrices, Linear Algebra Appl. 432 (2010), no. 2, 366-368.

4. K. Balachandran and J.Y. Park, Existence of solutions and controllability of nonlinear integrodifferential systems in Banach spaces, Math. Problems in Engineering 2 (2003), 6579.

5. C. Bennett and R. Sharpley, Interpolation of Operators, Academic Press, Inc., Orlando, 1988.

6. D. Chen and Y. Zhang, On the spectral radius of Hadamard products of nonnegative matrices, Banach J. Math. Anal. 9 (2015), no. 2, 127-133.

7. G.P. Curbera and W.J. Ricker, Compactness properties of Sobolev imbeddings for rearrangement invariant norms, Transactions AMS 359 (2007), 1471-1484.

8. P. Degond, J.-G. Liu and C. Ringhofer, Evolution of the distribution of wealth in an economic environment driven by local Nash equilibria, Journal of Statistical Physics 154 (2014), 751-780.

9. R. Drnovšek and A. Peperko, Inequalities for the Hadamard weighted geometric mean of positive kernel operators on Banach function spaces, Positivity 10 (2006), 613-626.

10. R. Drnovšek and A. Peperko, On the spectral radius of positive operators on Banach sequence spaces, Linear Algebra Appl. 433 (2010), 241-247. 
11. R. Drnovšek and A. Peperko, Inequalities on the spectral radius and the operator norm of Hadamard products of positive operators on sequence spaces, to appear in Banach J. Math. Anal. (2016).

12. L. Elsner, D. Hershkowitz and A. Pinkus, Functional inequalities for spectral radii of nonnegative matrices, Linear Algebra Appl. 129 (1990), 103-130.

13. L. Elsner, C.R. Johnson and J.A. Dias Da Silva, The Perron root of a weighted geometric mean of nonnegative matrices, Linear Multilinear Algebra 24 (1988), 1-13.

14. R.A. Horn and F. Zhang, Bounds on the spectral radius of a Hadamard product of nonnegative or positive semidefinite matrices, Electron. J. Linear Algebra 20 (2010), 90-94.

15. Z. Huang, On the spectral radius and the spectral norm of Hadamard products of nonnegative matrices, Linear Algebra Appl. 434 (2011), 457-462.

16. K. Jörgens, Linear integral operators, Surveys and Reference Works in Mathematics 7, Pitman Press, 1982.

17. J. Lafferty and G. Lebanon, Diffusion kernels on statistical manifolds, Journal of Machine Learning Research 6 (2005), 129-163.

18. A. Peperko, Inequalities for the spectral radius of non-negative functions, Positivity $\mathbf{1 3}$ (2009), 255-272.

19. A. Peperko, On the functional inequality for the spectral radius of compact operators, Linear Multilinear Algebra 59 (2011), no. 4, 357-364.

20. A. Peperko, Bounds on the generalized and the joint spectral radius of Hadamard products of bounded sets of positive operators on sequence spaces, Linear Algebra Appl. 437 (2012), 189-201.

21. A. Peperko, Bounds on the joint and generalized spectral radius of Hadamard geometric mean of bounded sets of positive kernel operators, (2016), submitted.

22. A.R. Schep, Bounds on the spectral radius of Hadamard products of positive operators on $l_{p}$-spaces, Electronic J. Linear Algebra 22, (2011), 443-447.

23. A.R. Schep, Corrigendum for "Bounds on the spectral radius of Hadamard products of positive operators on $l_{p}$-spaces", (2011), preprint.

24. A.C. Zaanen, Riesz Spaces II, North Holland, Amsterdam, 1983.

25. X. Zhan, Unsolved matrix problems, Talk given at Advanced Workshop on Trends and Developments in Linear Algebra, ICTP, Trieste, Italy, July 6-10, 2009.

1 Faculty of Mechanical Engineering, University of Luubluana, AškerČeva 6 , SI-1000 LuUbluana, Slovenia;

${ }^{2}$ Institute of Mathematics, Physics, and Mechanics, Jadranska 19, SI-1000 LjublJANA, Slovenia.

E-mail address: aljosa.peperko@fmf.uni-lj.si; aljosa.peperko@fs.uni-lj.si 